\title{
Spontaneous Polariton Currents in Periodic Lateral Chains
}

\author{
A. V. Nalitov, ${ }^{1}$ T. C. H. Liew, ${ }^{2}$ A. V. Kavokin, ${ }^{1,3,4,5}$ B. L. Altshuler, ${ }^{6}$ and Y. G. Rubo ${ }^{7,8}$ \\ ${ }^{1}$ School of Physics and Astronomy, University of Southampton, Southampton SO17 1BJ, United Kingdom \\ ${ }^{2}$ Division of Physics and Applied Physics, School of Physical and Mathematical Sciences, \\ Nanyang Technological University, Singapore 637371, Singapore \\ ${ }^{3}$ CNR-SPIN, Viale del Politecnico 1, I-00133 Rome, Italy \\ ${ }^{4}$ Spin Optics Laboratory, St. Petersburg State University, St. Petersburg 198504, Russia \\ ${ }^{5}$ Russian Quantum Center, 100 Novaya Street, Skolkovo, Moscow Region 143025, Russia \\ ${ }^{6}$ Physics Department, Columbia University, New York, New York 10027, USA \\ ${ }^{7}$ Instituto de Energías Renovables, Universidad Nacional Autónoma de México, \\ Temixco, Morelos 62580, Mexico \\ ${ }^{8}$ Center for Theoretical Physics of Complex Systems, Institute for Basic Science (IBS), \\ Daejeon 34051, Republic of Korea
}

(Received 17 February 2017; published 11 August 2017)

\begin{abstract}
We predict spontaneous generation of superfluid polariton currents in planar microcavities with lateral periodic modulation of both the potential and decay rate. A spontaneous breaking of spatial inversion symmetry of a polariton condensate emerges at a critical pumping, and the current direction is stochastically chosen. We analyze the stability of the current with respect to the fluctuations of the condensate. A peculiar spatial current domain structure emerges, where the current direction is switched at the domain walls, and the characteristic domain size and lifetime scale with the pumping power.
\end{abstract}

DOI: 10.1103/PhysRevLett.119.067406

Being weakly interacting composite bosons, excitonpolaritons undergo Bose-Einstein condensation (BEC) $[1,2]$ and may exhibit superfluid behavior [3-5]. Its striking manifestation, persistent, frictionless polariton currents may be used for information exchange between optical logical devices [6,7]. Like atomic or molecular counterparts, polariton superfluids also sustain quantized vortices $[5,8]$ and half vortices [9-11]. Polariton transport free of backscattering may be as well realized in the linear regime at the edges of polariton topological insulators [12-14]. In all cases, strong light-matter coupling plays a crucial role, as it supplements light cavity photons with a strong nonlinearity and provides a strong magneto-optical interaction.

Contrary to the cold atom systems, cavity polaritons are characterized by finite lifetime, limited by the photon escape from the cavity. Formed as a result of the compensation of this dissipation by continuous pumping from exciton reservoirs, the polariton condensates are thus out of thermal equilibrium. In the case of nonresonant optical or electric pumping, the quantum coherence of the condensate is formed spontaneously. For sufficiently fast polariton thermalization, the condensate is formed in the single particle ground state in full analogy with the BEC. However, polaritons with a slow energy relaxation can choose an excited single-particle state for macroscopic occupation [15-17]. Such a state is usually degenerate. Moreover, when single-particle states possess different lifetimes, the interaction between polaritons can lead to condensation into specific many-particle states with spontaneously broken symmetries such as the time-reversal and parity symmetries [18]. In this weak lasing state, the system is stabilized by the repulsive polariton-polariton interactions rather than the reservoir depletion (gainsaturation nonlinearity). The combined effect of interactions and gain saturation extends the stability of weak lasing states to high excitation powers [19]. These states can be easily manipulated and switched experimentally [20,21].

Polariton condensates are commonly described in the mean field approximation with non-Hermitian Hamiltonians accounting for both decay and external pumping. In this Letter, we consider a lateral periodic complex potential for polaritons in planar microcavities or microwires similar to those realized in Refs. [15,22,23]. Its imaginary part, corresponding to spatially dependent polariton decay rate, is determined by the spatial modulation of the cavity quality factor. In turn, the real part of the potential may be realized with a spatial quantization energy modulation of either the photonic or excitonic component. Regardless of realization of this potential, the single-polariton mode with the longest lifetime turns out to be at the edge of the lowest energy minigap. Assuming the feed for all Bloch wave modes close to the bottom of the polariton dispersion being equal, this mode has the lowest lasing threshold.

In the case of in-phase modulation of the real and imaginary parts of the potential, a second threshold emerges due to the repulsive interaction. While below this threshold, the condensate order parameter period coincides with the modulation lattice constant, crossing it results in abrupt period doubling. There are two degenerate double period condensate states connected by the lattice translation spontaneous symmetry breaking. In terms of the two mode 
approximation, the transition is described as an admixture of the ground polariton state having an intermediate lifetime to the macroscopically occupied second band bottom state having the longest lifetime [22].

What happens if the modulations of the real and imaginary parts of the potential have opposite phases? In this case, the lowest threshold corresponds to the polariton mode at the top of the lowest miniband rather than at the bottom of the second miniband. The repulsive interaction blueshifts the initial condensate, causing the dynamical admixture of the second band bottom state. As we show below, there appears a nontrivial (neither 0 nor $\pi$ ) phase difference between these two states constituting the condensate, which manifests itself in a nonzero net polariton current.

It should be noted, that apart from some similarity, the formation of a spontaneous current is different in origin from the Kibble-Zurek mechanism of vorticity [24]. The polariton condensation does not follow the standard Kibble-Zurek scenario [25,26], and the current does not appear because of the nonadiabaticity of a transition. While nonzero current is a general feature of nonlinear Bloch solutions [27], in this Letter, we show that in the present case, there is no stationary condensate state without the flux, and this flux is not quantized.

We solve the Gross-Pitaevskii equation (GPE) for the condensate wave function taking into account two types of nonlinearities, stemming from polariton repulsion and reservoir depletion $(\hbar=1)$,

$\mathrm{i} \frac{\partial \Psi}{\partial t}=\left[-\frac{1}{2 m} \frac{\partial^{2}}{\partial x^{2}}+U+\frac{\mathrm{i}}{2}\left(W-\frac{\eta}{2}\left\langle|\Psi|^{2}\right\rangle\right)+\alpha|\Psi|^{2}\right] \Psi$.

Here, $m$ is the polariton effective mass and $\alpha$ is the interaction constant. The pumping power $W$, determined by the reservoir population, is locally reduced due to its depletion, which is proportional to the condensate density averaged over the unit cell with the prefactor $\eta / 2$. This nonlinearity can be obtained as a result of the exclusion of the equation for reservoir density [28]. The averaging describes washing out the spatial inhomogeneities of the reservoir density by the exciton diffusion and excludes unphysical solutions with a periodic modulation of the reservoir density. We consider a lateral complex periodic potential $U(x)$ for polaritons, shown in Fig. 1(a),

$U(x)= \begin{cases}-\frac{1}{2}(\tilde{\varepsilon}+\mathrm{i}(\Gamma-\tilde{\gamma})), & |x-n a|<\frac{1}{4} a, \\ \frac{1}{2}(\tilde{\varepsilon}-\mathrm{i}(\Gamma+\tilde{\gamma})), & \frac{1}{4} a<|x-n a|<\frac{1}{2} a,\end{cases}$

where $n$ spans all integers.

The Kronig-Penney model [29] for a polariton in such a potential yields the band structure [Fig. 1(b)], where the longest (shortest) lifetime characterizes the lowest-band top (the second-band bottom) state, which we denote as $S(A)$.

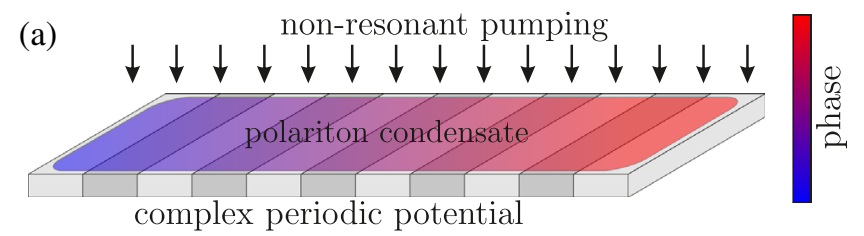

(b)
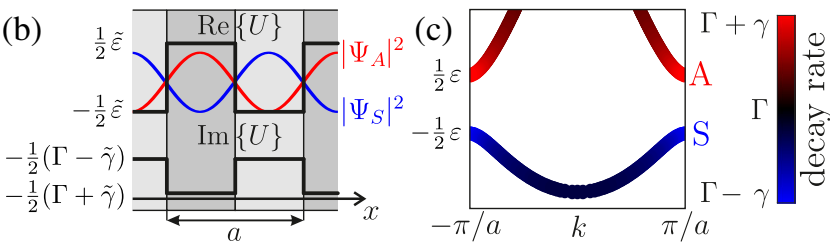

FIG. 1. (a) Sketch of the proposal: polariton current of spontaneously chosen direction flows in a condensate fed by a symmetric homogenous pumping. (b) Spatial distribution of real and imaginary parts of the periodic potential $U(x)$ and distribution of the first and second band wave functions at the Brillouin zone edge. (c) Complex polariton band structure in the KronigPenney model. The decay rate is shown with color. The fastest (slowest) decay rate characterizes $A(S)$ mode.

In the nearly free particle approximation, the two polariton modes $\Psi_{S} \propto \cos \left(k_{0} x\right)$ and $\Psi_{A} \propto \sin \left(k_{0} x\right)$ with $k_{0}=\pi / a$ are separated by the energy band gap $\varepsilon=2 \tilde{\varepsilon} / \pi$ and decay at rates $\Gamma-\gamma$ and $\Gamma+\gamma$, respectively, where $\gamma=2 \tilde{\gamma} / \pi$.

Assuming slow polariton thermalization and equal feeding of the modes from the reservoir, one should expect the longest lifetime mode $(S)$ to cross the lasing threshold first with increasing pumping power. With further growth of the condensate population, the repulsive interaction blueshifts the condensate and eventually leads to admixture of the second band bottom (A) state. We project Eq. (1) onto the plane wave two-mode basis and search for the solution in the form $\Psi=\psi_{+} \exp \left(+\mathrm{i} k_{0} x\right)+\psi_{-} \exp \left(-\mathrm{i} k_{0} x\right)$. Assuming that the envelopes $\psi_{ \pm}$are smooth on the scale of the lattice parameter $a$, we neglect second spatial derivatives of $\psi_{ \pm}$ and obtain

$\left[\frac{\partial}{\partial t} \pm c \frac{\partial}{\partial x}+\frac{g(s)}{2}+\frac{\mathrm{i} \alpha}{2}\left(3 s \mp s_{z}\right)\right] \psi_{ \pm}=\frac{\gamma+\mathrm{i} \varepsilon}{2} \psi_{\mp}$.

Here, $\quad c=\pi / m a, \quad g(s)=\eta s-w \quad$ with $\quad w=W-\Gamma$, $s=\left(\left|\psi_{+}\right|^{2}+\left|\psi_{-}\right|^{2}\right) / 2$, and $s_{z}=\left(\left|\psi_{+}\right|^{2}-\left|\psi_{-}\right|^{2}\right) / 2$. In what follows, we first find spatially homogeneous solutions

$$
\psi_{ \pm}(x, t) \equiv \psi_{ \pm}(t)=\sqrt{s \pm s_{z}} e^{-\mathrm{i}(\Omega t \pm \phi)},
$$

with time independent $s, s_{z}$, the phase shift $\phi$, and the emission frequency $\Omega$ (counted from the middle of the gap). Then, we study their stability with respect to small spatially nonhomogeneous fluctuations. See the Supplemental Material [30] for details of the derivations.

The first pair of solutions preserve the parity symmetry of Eqs. (3), i.e., $s_{z}=0$, which corresponds to the condensation in the single-particle $S$ state with $s=(w+\gamma) / \eta$ and $\phi=0$, and to the condensation in the $A$ state with 
$s=(w-\gamma) / \eta$ and $\phi=\pi / 2$. As expected, the $S$ mode solution has the lowest threshold pumping power $w=-\gamma$.

For the second pair of stationary solutions characterized by a nonzero $s_{z}$ and nontrivial phase difference $\phi$,

$s_{z}=-\frac{\varepsilon}{\gamma} s \tan (2 \phi), \quad \tan (2 \phi)= \pm \sqrt{\frac{\gamma^{2}-g(s)^{2}}{\varepsilon^{2}+g(s)^{2}}}$,

we obtain for the population $s$ and emission frequency $\Omega$,

$$
\varepsilon \alpha s g(s)=\gamma\left[\varepsilon^{2}+g(s)^{2}\right], \quad \Omega=\frac{1}{2}\left[3 \alpha s-\frac{\varepsilon \gamma}{g(s)}\right] .
$$

The first equation in (6) has two roots for the condensate population $s$. However, complemented with restrictions $s>0$ and $0<g(s)<\gamma$, it has two branches of solutions for pumping powers above the critical point

$$
w_{c}=\eta \frac{\gamma^{2}+\varepsilon^{2}}{\varepsilon \alpha}-\gamma,
$$

if $\alpha / \eta<(\gamma / \varepsilon)-(\varepsilon / \gamma)$ and a single branch of solutions otherwise. These two regimes correspond to subcritical and supercritical pitchfork bifurcations, respectively [31]. We refer to them as two transitions of types I and II, respectively, in an analogy to phase transitions of the first and the second order. Note that the condition $0<g(s)<\gamma$ implies that these solutions correspond to intermediate condensate populations between populations of the symmetric $S$ and antisymmetric $A$ solutions.

The branches of fixed points for both cases are shown in Fig. 2; $s(w)$ shows the normalized condensate population, while the nonzero population imbalance $s_{z}(w)$ defines the polariton current flowing in the condensate $j=2 k_{0} s_{z} / \mathrm{m}$. For $A$ and $S$ state solutions, shown in red and blue, respectively, there is no current, and populations are linear in the pumping power. The nonzero current is a property of the other pair of branches, plotted in black. The two solutions have the same population, but the current directions are opposite. It is important to note that the polariton density for the current solutions is lower than the maximal possible density achieved at the unstable state $S$. This is characteristic for the weak lasing regime, where the losses in the polariton system are adjusted to compensate the gain rather than minimized.

Figure 2 also indicates the stability of the solutions with respect to homogeneous fluctuations; the unstable branches are shown with dotted lines. While the $A$ state is always unstable, the $S$ state solution is stable at $s<s_{c}=$ $\left(\varepsilon^{2}+\gamma^{2}\right) / \alpha \varepsilon$, below the critical pumping value $w_{c}$. The symmetry breaking solutions, on the contrary, are stable above the critical pumping in the case of a type II bifurcation. For a type I with two nontrivial solutions for $s(w)$, the lower branch is stable, while the upper one is unstable. The critical point $w=w_{c}$ is thus a pitchfork bifurcation of a cusp catastrophe. It is supercritical for
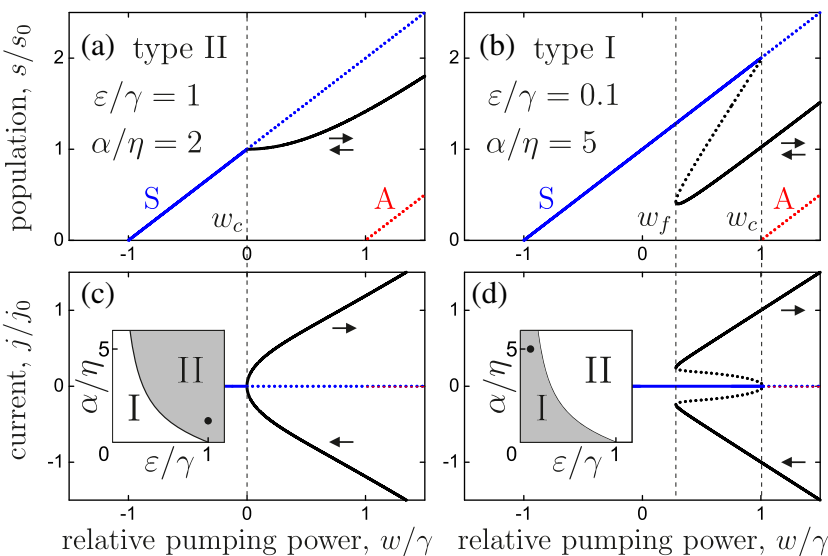

FIG. 2. (a),(b) Condensate population dependence on the pumping power. (c),(d) Imbalance of populations $s_{z}$ defining the value of the net polariton current. Uniformly stable (unstable) stationary solutions are plotted with solid (dotted) lines. Blue and red lines correspond to symmetry conserving condensates at $S$ and $A$ polariton modes. The two sets of parameters correspond to the presence (type I) and absence (type II) of a tristability region between the $S$ state solution and the pair of symmetry breaking solutions. In the former case, two hysteresis loops emerge. The lower two plots are zoomed to the pitchfork and saddlenode bifurcation points $w_{c}$ and $w_{f}$. Inset: phase transition type depending on $\alpha / \eta$ and $\gamma / \varepsilon$.

type II and subcritical for type I. The latter is accompanied with a region of tristability below the critical point. It is limited from below with a saddle-node bifurcation at $w=w_{f}=2 \sqrt{\gamma \eta(\gamma \eta-\varepsilon \alpha)} / \alpha<w_{c}$. The tristability region corresponds to hysteresis loops between the symmetric $S$ state solution and the pair of symmetry breaking solutions. The sign of $s_{z}$ and thus the direction of the polariton current $j$ is spontaneously chosen by the system once the condensate passes the critical point. Its value continuously grows from zero in the supercritical case and appears abruptly in the subcritical one. On the way back along a randomly chosen hysteresis loop, the current abruptly disappears at the saddle-node bifurcation point $w=w_{c}$.

To study the stability with respect to inhomogeneous phase and population fluctuations, we calculate the elementary excitation spectra. In the standard way, we linearize Eq. (3) with respect to a plane wave perturbation

$$
\delta \psi_{ \pm}(x, t)=e^{-\mathrm{i} \Omega t}\left(u_{ \pm} e^{\mathrm{i} k x+\lambda t}+v_{ \pm}^{*} e^{-\mathrm{i} k x+\lambda^{*} t}\right)
$$

of the spatially uniform solutions described above.

The dispersion of the real and imaginary parts of the Lyapunov exponent $\lambda(k)$ for the nonzero current solution is plotted in Fig. 3 for different regimes. Here, we consider uniformly stable solutions, characterized by three modes with $\operatorname{Re} \lambda(0)<0$ and one Goldstone mode with $\lambda(0)=0$. The Goldstone mode appears due to the irrelevance to the global shift of the total phase of the condensate $\Phi$. 

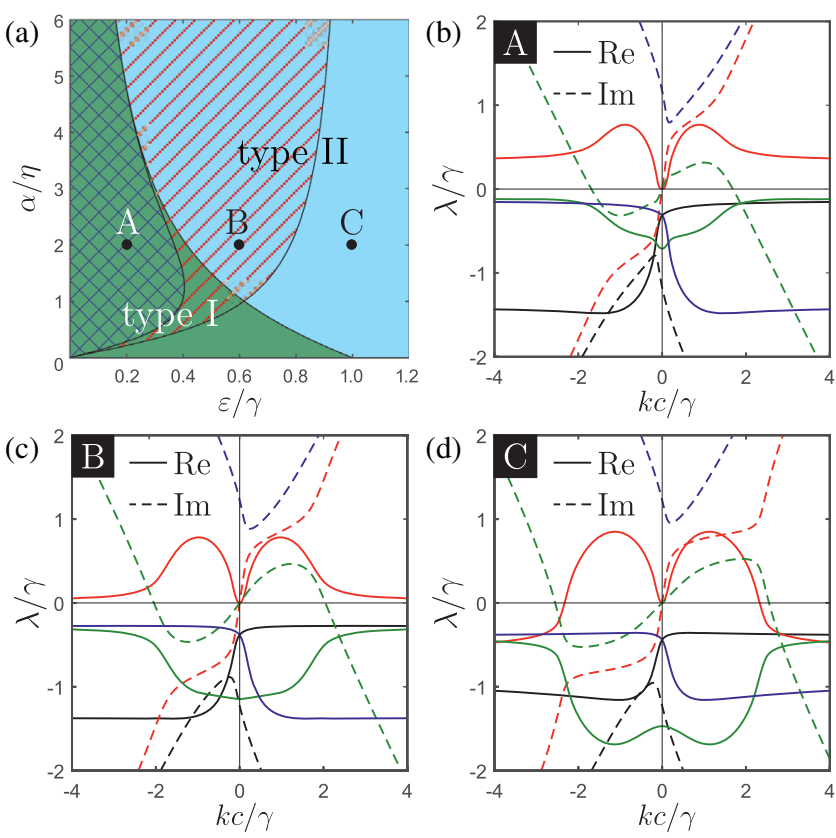

FIG. 3. (a) Stability diagram. Type I and II transitions are shown with green and blue colors, respectively. Regimes of current stability with respect to short wavelength fluctuations are shown with hatching: the blue double hatched area corresponds to the unstable regime; the red single hatched area covers the parameter space where the current is stable in a range of pumping powers; the area free of hatching corresponds to the stable current regime. (b)-(d) The Lyapunov spectra of condensate current solutions. Solid and dashed lines show the real and imaginary parts, respectively. Parameters correspond to the black dots in the panel (a). The pumping power is slightly above the critical point.

The short wavelength limit analysis provides an important condition of the applicability of the two-mode approximation (3). Depending on the parameters, there are three regimes, illustrated by Fig. 3(a). For a given nonlinearity relation $\alpha / \eta$, low values of $\varepsilon / \gamma$ correspond to the instability of the symmetry breaking condensate in the short wavelength limit. In this domain, the system should exhibit either period doubling bifurcations [22] or strongly chaotic behavior. There is an intermediate regime where the spontaneous current condensate is stable in a certain range of pumping powers. However, in the most realistic case of a large $\varepsilon / \gamma$, the spontaneous current solutions are stable with respect to short wavelength fluctuations and are well described by Eqs. (3). Note that for the long wavelength fluctuations, the condition $k a \ll 1$ is well satisfied. The two parameters defining the short wavelength stability, $\alpha / \eta$ and $\varepsilon / \gamma$ depend on the system design: the interaction nonlinearity $\alpha$ may be controlled by polariton lateral confinement (etched microcavity width), while $\varepsilon$ and $\gamma$ are independently tunable through periodic modulation of the cavity photonic mode energy and broadening.

In the case that the condensate is stable with respect to short wavelength fluctuations, there is still a region of positive Lyapunov exponents, as it is seen from Fig. 3(d). We note that this result does not follow from the MerminWagner theorem [32], although the latter also forbids long-range order in 1D. A condensate in a long enough microcavity chain thus falls apart and is expected to transform into a polariton current domain structure. The characteristic domain size $l_{d} \sim c / w$, deduced from the extremum position of the excitation spectrum imaginary part, as well as its characteristic lifetime $\tau_{d} \sim 1 / w$, obtained as the inverse extremum value, scales as the inverse occupation number of the condensate. Hence, the domain wall characteristic speed $v_{d} \sim l_{d} / \tau_{d} \approx c$ is independent on the pumping power.

On the other hand, a finite system with periodic boundary conditions, such as a microcavity ring chain, may support a global bifurcation towards a polariton condensate with a spontaneously chosen and persistent circular current. This is possible in the case of short wavelength stability in a range of low pumping powers. The upper boundary of this range is determined by the cutoff fluctuation wave vector $k_{c}$ defined by $\operatorname{Re} \lambda\left(k_{c}\right)=0$ [see red curve in Fig. 3(d)]. The persistent current is possible in the ring with radius $R<k_{c}^{-1}$. We note
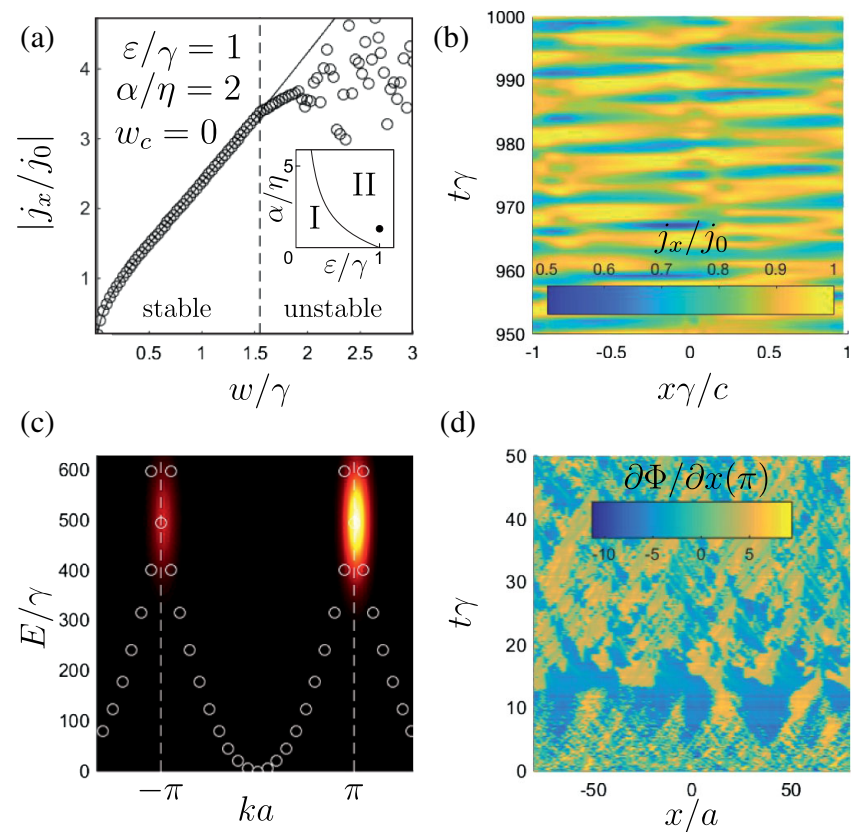

(d)

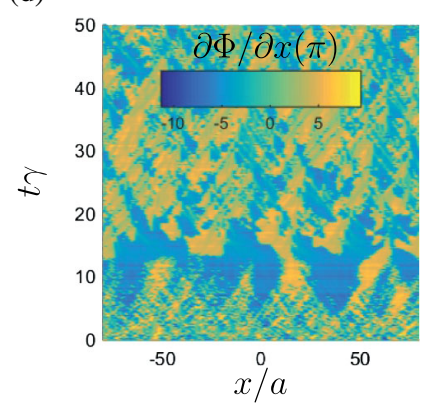

FIG. 4. (a), (b) Numerical simulation in the two-mode approximation. (a) Absolute value of the spatially averaged current vs pumping power. The system finite size defines the instability onset point. Parameters correspond to the type II transition with the finite system size set by $-c \leq x \gamma \leq c$. (b) Spatial and temporal dependence of the polariton current in the unstable regime (for $w=2.1 \gamma$ ): domain structure emerges in an initially homogeneous condensate. (c),(d) Full numerical simulation of GPE with a periodic complex potential. (c) Condensate dispersion demonstrating spatial asymmetry. The intensity has been broadened in energy and momentum for visualization. Circles denote the bare dispersion, quantized by the finite system size. Parameters: $w=\gamma,-a \leq x \leq a$. (d) Spatial and temporal dependence of local phase gradient in the unstable regime (given by a larger system size $-80 a \leq x \leq 80 a$ ), with chaotic evolution of domain structure. 
that this mechanism of formation of stable polariton currents in modulated polariton rings is different to that in unmodulated polariton rings, in which high angular momentum states were shown to be unstable [33]. Here, the circular polariton current is formed spontaneously and is not inherited from an optical pump.

Equations (3) can be solved numerically, by propagating in time from an initial random noisy state. The results of this numerics for the type-II transition are shown in Fig. 4(a). A stable current is formed spontaneously from the initial noise when the pump intensity is below the critical value set by the finite system size. Above the critical pump intensity, the system breaks up forming oscillating domains [see Fig. 4(b)]. For very large pump intensities, a chaotic state forms with strong fluctuations in the spatially averaged current.

The spontaneous currents can also be obtained by a direct numerical solution of the original GPE (1), without the two-mode approximation (3). Here, we add a Langevin noise term [17], which serves both as an initial seed for the condensate and a test of its stability to fluctuations. Using type II parameters, we find preferential condensation in the $k=+k_{0}$ state [see Fig. 4(c)]. This state is further characterized by a relatively small and spatially uniform phase gradient (not shown), which is stable in time despite the presence of noise. Repeating the calculation revealed random selection of the $\pm k_{0}$ states, with equal probability. Finally, by increasing the system size, the spontaneous currents become unstable leading to the formation of chaotic domains, characterized by different phase gradients, which evolve spatiotemporally.

In summary, we considered polariton condensation in microcavities with a potential and decay rate periodically modulated in space. Our analysis suggests that such systems undergo a spontaneous symmetry breaking and the formation of polariton currents. We identify the critical conditions for this effect to emerge and produce the phase diagram showing type I and type II transition boundaries. For large systems, oscillating domains of counterpropagating currents are predicted. For systems smaller than the characteristic domain size, e.g., polariton rings, the spontaneously formed currents are stable and survive in the presence of spatiotemporal noise. See the Supplemental Material [34] for discussion of the experimental realization of the effect.

This work has been supported in part by CONACYT (Mexico) Grant No. 251808, by IBS-R024-D1, and the Ministry of Education (MOE) (Singapore) Grant No. 2015T2-1-055. AK acknowledges the support from the Russian Foundation for Basic Research, Grant No. 15-59-30406 PT, DFG ICRC Project No. TR 160, and the EPSRC Established Career Fellowship in Quantum Polaritonics.

[1] R. Balili, V. Hartwell, D. Snoke, L. Pfeiffer, and K. West, Science 316, 1007 (2007).
[2] J. Kasprzak, M. Richard, S. Kundermann, A. Baas, P. Jeambrun, J. M. J. Keeling, F. M. Marchetti, M. H. Szymańska, R. André, J. L. Staehli, V. Savona, P. B. Littlewood, B. Deveaud, and L. S. Dang, Nature (London) 443, 409 (2006).

[3] A. Amo, J. Lefrère, S. Pigeon, C. Adrados, C. Ciuti, I. Carusotto, R. Houdré, E. Giacobino, and A. Bramati, Nat. Phys. 5, 805 (2009).

[4] A. Amo, D. Sanvitto, F. P. Laussy, D. Ballarini, E. del Valle, M. D. Martin, A. Lemaître, J. Bloch, D. N. Krizhanovskii, M. S. Skolnick, C. Tejedor, and L. Viña, Nature (London) 457, 291 (2009).

[5] D. Sanvitto, F. M. Marchetti, M. H. Szymańska, G. Tosi, M. Baudisch, F. P. Laussy, D. N. Krizhanovskii, M. S. Skolnick, L. Marrucci, A. Lemaître, J. Bloch, C. Tejedor, and L. Viña, Nat. Phys. 6, 527 (2010).

[6] T. C. H. Liew, A. V. Kavokin, and I. A. Shelykh, Phys. Rev. Lett. 101, 016402 (2008).

[7] T. C. H. Liew, A. V. Kavokin, T. Ostatnický, M. Kaliteevski, I. A. Shelykh, and R. A. Abram, Phys. Rev. B 82, 033302 (2010).

[8] K. G. Lagoudakis, M. Wouters, M. Richard, A. Baas, I. Carusotto, R. André, L. S. Dang, and B. Deveaud-Plédran, Nat. Phys. 4, 706 (2008).

[9] Y. G. Rubo, Phys. Rev. Lett. 99, 106401 (2007).

[10] K. G. Lagoudakis, T. Ostatnický, A. V. Kavokin, Y. G. Rubo, R. André, and B. Deveaud-Plédran, Science 326, 974 (2009).

[11] R. Hivet, H. Flayac, D. D. Solnyshkov, D. Tanese, T. Boulier, D. Andreoli, E. Giacobino, J. Bloch, A. Bramati, G. Malpuech, and A. Amo, Nat. Phys. 8, 724 (2012).

[12] A. V. Nalitov, D. D. Solnyshkov, and G. Malpuech, Phys. Rev. Lett. 114, 116401 (2015).

[13] T. Karzig, C.-E. Bardyn, N.H. Lindner, and G. Refael, Phys. Rev. X 5, 031001 (2015).

[14] C.-E. Bardyn, T. Karzig, G. Refael, and T. C. H. Liew, Phys. Rev. B 91, 161413 (2015).

[15] C. W. Lai, N. Y. Kim, S. Utsunomiya, G. Roumpos, H. Deng, M. D. Fraser, T. Byrnes, P. Recher, N. Kumada, T. Fujisawa, and Y. Yamamoto, Nature (London) 450, 529 (2007).

[16] N. Y. Kim, K. Kusudo, C. Wu, N. Masumoto, A. Löffler, S. Höfling, N. Kumada, L. Worschech, A. Forchel, and Y. Yamamoto, Nat. Phys. 7, 681 (2011).

[17] K. Winkler, O. A. Egorov, I. G. Savenko, X. Ma, E. Estrecho, T. Gao, S. Müller, M. Kamp, T. C. H. Liew, E. A. Ostrovskaya, S. Höfling, and C. Schneider, Phys. Rev. B 93, 121303 (2016).

[18] I. L. Aleiner, B. L. Altshuler, and Y. G. Rubo, Phys. Rev. B 85, 121301 (2012).

[19] H. Ohadi, A. Dreismann, Y. G. Rubo, F. Pinsker, Y. del ValleInclan Redondo, S. I. Tsintzos, Z. Hatzopoulos, P. G. Savvidis, and J. J. Baumberg, Phys. Rev. X 5, 031002 (2015).

[20] H. Ohadi, Y. del Valle-Inclan Redondo, A. Dreismann, Y. G. Rubo, F. Pinsker, S. I. Tsintzos, Z. Hatzopoulos, P. G. Lagoudakis, and J. J. Baumberg, Phys. Rev. Lett. 116, 106403 (2016).

[21] A. Dreismann, H. Ohadi, Y. del Valle-Inclan Redondo, R. Balili, Y. G. Rubo, S. I. Tsintzos, G. Deligeorgis, Z. Hatzopoulos, P. G. Savvidis, and J. J. Baumberg, Nat. Mater. 15, 1074 (2016). 
[22] L. Zhang, W. Xie, J. Wang, A. Poddubny, J. Lu, Y. Wang, J. Gu, W. Liu, D. Xu, X. Shen, Y. G. Rubo, B. L. Altshuler, A. V. Kavokin, and Z. Chen, Proc. Natl. Acad. Sci. U.S.A. 112, E1516 (2015).

[23] T. Gao, E. Estrecho, G. Li, O. A. Egorov, X. Ma, K. Winkler, M. Kamp, C. Schneider, S. Höfling, A. G. Truscott, and E. A. Ostrovskaya, Phys. Rev. Lett. 117, 097403 (2016).

[24] W. H. Zurek, Nature (London) 317, 505 (1985).

[25] M. Matuszewski and E. Witkowska, Phys. Rev. B 89, 155318 (2014).

[26] D. D. Solnyshkov, A. V. Nalitov, and G. Malpuech, Phys. Rev. Lett. 116, 046402 (2016).

[27] I. Y. Chestnov, A. V. Yulin, A. P. Alodjants, and O. A. Egorov, Phys. Rev. B 94, 094306 (2016).

[28] J. Keeling and N. G. Berloff, Phys. Rev. Lett. 100, 250401 (2008).

[29] G. Bastard, Wave Mechanics Applied to Semiconductor Heterostructures (Les Éditions de Physique, Les Ulis, 1988).

[30] See Supplemental Material http://link.aps.org/supplemental/ 10.1103/PhysRevLett.119.067406 for detailed derivations and stability analysis, which includes Refs. [5,10,15,28,3538].
[31] S. H. Strogatz, Nonlinear Dynamics and Chaos (Perseus Books, New York, 1994).

[32] P. C. Hohenberg, Phys. Rev. 158, 383 (1967).

[33] G. Li, M. D. Fraser, A. Yakimenko, and E. A. Ostrovskaya, Phys. Rev. B 91, 184518 (2015).

[34] See Supplemental Material http://link.aps.org/supplemental/ 10.1103/PhysRevLett.119.067406 for discussion of the experimental realization of the effect, which includes Refs. [5,10,15,28,35-38].

[35] K. G. Lagoudakis, F. Manni, B. Pietka, M. Wouters, T. C. H. Liew, V. Savona, A. V. Kavokin, R. André, and B. DeveaudPlédran, Phys. Rev. Lett. 106, 115301 (2011).

[36] G. Nardin, K. G. Lagoudakis, B. Pietka, F. Morier-Genoud, Y. Léger, and B. Deveaud-Plédran, Phys. Rev. B 82, 073303 (2010).

[37] M. M. Glazov, H. Ouerdane, L. Pilozzi, G. Malpuech, A. V. Kavokin, and A. D'Andrea, Phys. Rev. B 80, 155306 (2009).

[38] A. Askitopoulos, T. C. H. Liew, H. Ohadi, Z. Hatzopoulos, P. G. Savvidis, and P. G. Lagoudakis, Phys. Rev. B 92, 035305 (2015). 